\title{
EAS age and energy determinations through the study of the LDF in the first few meters from the core with the ARGO-YBJ experiment
}

\author{
P. Bernardini, A. D’Amone, I. De Mitri*, G. Marsella, A. Surdo \\ Dipartimento di Matematica e Fisica "E. De Giorgi" - Università del Salento \\ and Istituto Nazionale di Fisica Nucleare (INFN) - Sezione di Lecce \\ Via per Arnesano, I-73100, Lecce, Italy \\ E-mail: ivan.demitri@le.infn.it
}

for the ARGO-YBJ Collaboration

The ARGO-YBJ experiment, a full coverage extensive air shower (EAS) detector located at high altitude (4300 $\mathrm{m}$ a.s.1.) in Tibet, China, has been operated with very high stability from the fall 2007 to the beginning of 2013. The array consisted of a carpet of about $7000 \mathrm{~m}^{2}$ Resistive Plate Chambers (RPCs) operated in streamer mode and equipped with both digital and analog readout, providing the measurement of particle densities up to few particles per $\mathrm{m}^{2}$, with single hit space and time resolutions better than $20 \mathrm{~cm}$ and $2 \mathrm{~ns}$, respectively. The unique detector features (full coverage, readout granularity, wide dynamic range, ..) and location (very high altitude) allowed a detailed study of the lateral distribution function (LDF) of particles at ground very close to the shower axis, in a wide interval of primary energies from few $\mathrm{TeV}$ up to and beyond the knee of the all-particle spectrum. The information collected in the first 10 meters from the shower has been shown to provide very effective tools for the determination of both energy and shower age. The shower age was shown to be correlated with the reconstructed LDF slope near the core, independently of the primary mass. This shower universality was then used to correct the detected truncated size at ground in order to have a mass independent energy estimator with a lognormal resolution at the level of 0.15 , getting better with energy. The details of the adopted procedure together with the evaluation of its uncertainties will be fully discussed.

The 34th International Cosmic Ray Conference,

30 July- 6 August, 2015

The Hague, The Netherlands

\footnotetext{
* Speaker.
} 


\section{Introduction}

The shower development stage in the atmosphere, the so-called longitudinal age, as observed at a fixed altitude (the detection one), depends on the energy of the interacting primary. For fixed energy, it depends on the primary nature: heavier primaries interact higher in the atmosphere, thus giving showers which, on average, reach their maximum size at a greater distance from the detector than a lighter primary of the same energy. For this reason, the combined use of shower energy and age estimations can ensure the sensitivity to the primary nature. On the other hand, the knowledge of the development stage of a detected shower, as expressed by the height of its maximum in the atmosphere, $X_{d m}\left(\mathrm{~g} / \mathrm{cm}^{2}\right)$, can allow to infer its energy, whatever the primary nature is.

An EAS array by itself cannot measure directly the shower development stage, through the determination of the depth of the shower maximum, $X_{\max }$ (as made for instance by fluorescence detectors). It can only measure the particle density distribution at ground as a function of the core distance (described through a lateral density function, LDF) and from the slope of this distribution get information on the longitudinal shower development. In fact, the detailed study of the lateral particle density profile at ground is expected to provide information on the longitudinal profile of the showers in the atmosphere, that is to estimate their development stage, or age.

Historically, it was shown that such lateral distribution (at least of "electrons"), as measured by a traditional sampling EAS array at core distances of the order of hundred meters, can be described by a LDF like the Nishimura-Kamata-Greisen (NKG) structure function [1, 2], with parameters reflecting the shower size, the detection altitude and the shower age. The age parameter determined in this way is usually referred to as lateral age [3, 4], since obtained from the LDF. It, in principle, coincides with the longitudinal age in particular for purely e.m. EASs, but, in an experimental context, they can in fact differ, since most showers come from hadrons and the two quantities are measured with completely different techniques. However, they must be strongly related.

\section{The ARGO-YBJ experiment}

The ARGO-YBJ detector is a full coverage extensive air shower (EAS) array made by a single layer of Resistive Plate Chambers (RPCs) operated in streamer mode, for $\gamma$-astronomy and cosmic rays (CR) studies [5]. The array, installed in the Cosmic Ray Laboratory of YanBaJing (Tibet, China) at an altitude of $4300 \mathrm{~m}$ above sea level (corresponding to a vertical atmospheric depth of about $606 \mathrm{~g} / \mathrm{cm}^{2}$ ), ran in its full configuration since November 2007 until February 2013. It is organized in 153 clusters of 12 RPCs each. Any single RPC is read out by ten $62 \times 56 \mathrm{~cm}^{2}$ pads, which are further divided into 8 strips, thus providing a larger particle counting dynamic range $[6,7]$. The signals coming from all the strips of a given pad are sent to the same channel of a multi-hit TDC. The whole system provides a single hit (pad) time resolution of $\sim 1.8 \mathrm{~ns}$, which, joined to the full coverage feature, allows a complete and detailed three-dimensional reconstruction of the shower front with unprecedented space-time resolution. A system for the RPC analog charge readout [8] from larger pads, each one covering half a chamber (the so called big pads, BP), has also been implemented and took data since January 2010. This actually extended the detector sensitivity range from about $10^{14} \mathrm{eV}$ up to about $10^{16} \mathrm{eV}$ for primary protons. The analog readout system has been operated with different gain scales (from G0 to G7, with increasing gains), which determine the threshold and the maximum number of particles that can be reliably measured on each BP. 

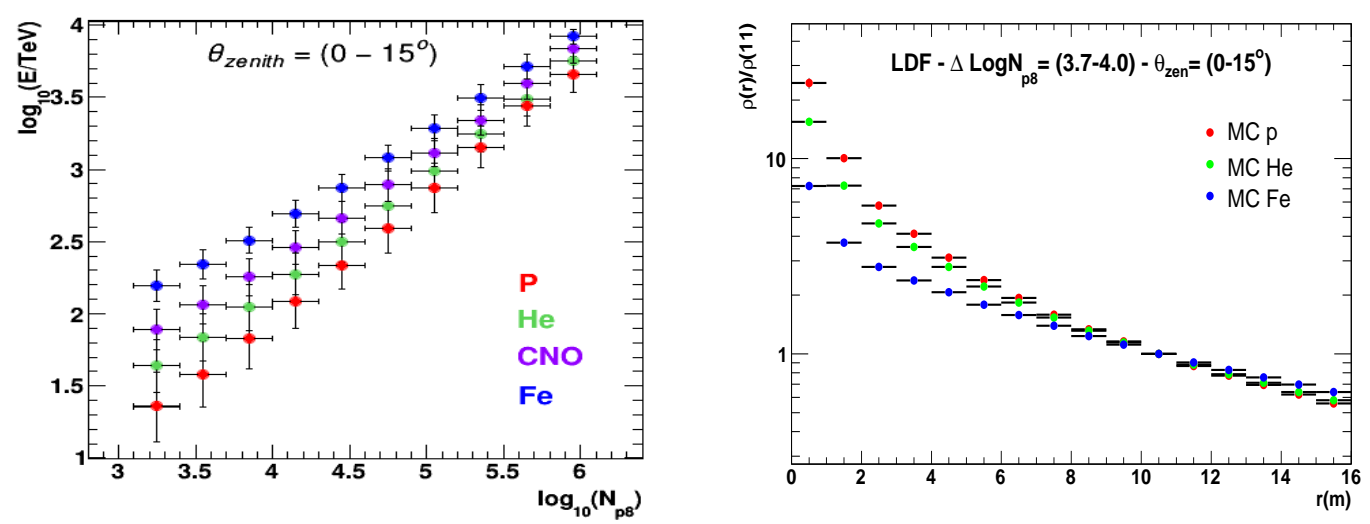

Figure 1: Left: Average primary energy for $\mathrm{p}, \mathrm{He}, \mathrm{CNO}$ group and Fe initiated $\mathrm{MC}$ showers for various intervals of $N_{p 8}$, the number of detected particles within $8 \mathrm{~m}$ from the shower axis. Vertical error bars are the RMSs of the energy distributions. Right: Reconstructed LDF of the detected particles around the shower axis for simulated $\mathrm{p}, \mathrm{He}$ and $\mathrm{Fe}$ initiated showers with $10^{3.7}<N_{p 8}<10^{4.0}$ and zenith angle less than $15^{\circ}$. The three distributions are here normalized to the particle density at $11 \mathrm{~m}$ of distance from the shower axis.

The highest gain scale G7 allows low density values to be measured down to few particles per $\mathrm{m}^{2}$, overlapping its dynamic range with the detector operated in 'digital mode', i.e. simply counting the number of fired strips, that saturates at about $20 / \mathrm{m}^{2}$. The other scales have decreasing gains (down to G0) and allow measuring ever increasing densities up to several $10^{4} / \mathrm{m}^{2}$ [8]. These features allow the study of the detailed structure of the particle density distribution at the observation level even very close to the shower axis.

\section{Monte Carlo simulations and data analysis}

For the analysis here presented, several air shower samples induced by proton, He, CNO elemental group and $\mathrm{Fe}$ primaries have been simulated, for a total amount of several millions events in the $\left(10^{12}-10^{16}\right) \mathrm{eV}$ energy range. The simulated showers were produced by using the CORSIKA code [9], with QGSJET-II.03 [10] as hadronic interaction model, and following the spectra as given in [11], with zenith angle $\theta<45^{\circ}$. With cores randomly sampled in a larger area (about ten times) than the detector surface, such showers have been given in input to a GEANT [12] based program fully simulating the detector structure and response (including the effects of time resolution, trigger logic, electronics noise, readout system, etc.). The Monte Carlo (MC) events triggering the analog system readout ( $\geq 73$ fired pads in a cluster) have then been processed by the same reconstruction program used for real data. The events were subsequently selected by requiring the core position, which is reconstructed with an accuracy of the order of $1 \mathrm{~m}$ or less, to be in a fiducial area of $64 \times 64 \mathrm{~m}^{2}$ around the detector center. This work was also restricted to events with reconstructed zenith angle $\theta<15^{\circ}$. The study of these MC events (see [13]) allowed to identify the truncated size $N_{p 8}$, defined as the number of particles detected within a distance of $8 \mathrm{~m}$ from the shower axis, as a suitable estimator of the primary CR energy E, since well correlated with E, not biased by effects due to finite detector size, nor dominated by shower to shower fluctuations. In Fig. 1, the correlation between $E$ and $N_{p 8}$ is shown for simulated proton, helium, CNO group and iron initiated showers. Several $N_{p 8}$ intervals have been chosen to select event samples corresponding to different primary energies. Clearly, the $N_{p 8}$ quantity is a mass dependent primary energy estimator. 

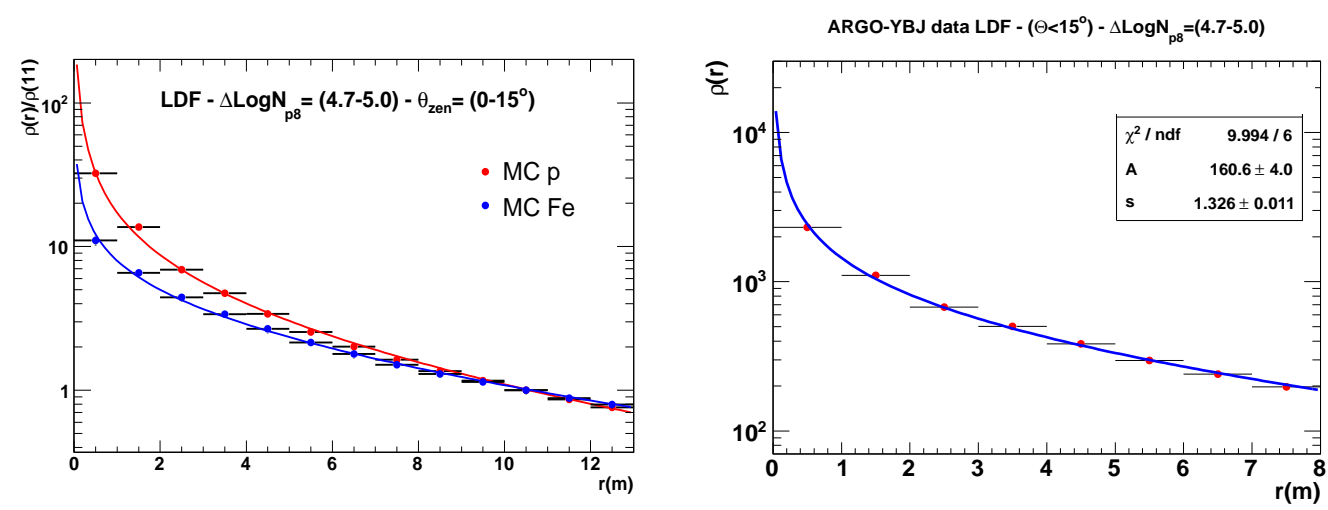

Figure 2: Left: Fits to the reconstructed LDFs of the detected particles around the axis for simulated proton and iron-nucleus induced showers with $10^{4.7}<N_{p 8}<10^{5.0}$, by the NKG-like function in Eq. 4.1. Right: Reconstructed LDF of the detected particles around the shower axis for ARGO-YBJ experimental data with $10^{4.7}<N_{p 8}<10^{5.0}$ and $\theta<15^{\circ}$. The superimposed fit with the NKG-like function is also shown.

\section{Parametrizations of the LDF}

As already stated in the Section 1, the basic idea is to get information on the shower development stage from the lateral density distribution structure around the shower axis. Thus, the average particle distributions starting from the reconstructed core have been studied in detail for each $N_{p 8}$ bin and, in the case of simulations, for different primary types. As an example, the Fig. 1 (right) shows the average lateral distribution of particles obtained for a sample of simulated proton induced shower events in the interval $\Delta N_{p 8}=10^{3.7}-10^{4.0}$, corresponding to an average energy $E_{p} \simeq 70 \mathrm{TeV}$, together with the average LDFs for He and Fe primaries in the same $\Delta N_{p 8}$ (corresponding to an average energy $E_{\mathrm{He}} \simeq 100 \mathrm{TeV}$ and $E_{\mathrm{Fe}} \simeq 300 \mathrm{TeV}$, respectively).

Various modifications of the NKG form were proposed as LDFs in order to optimize the function for reproducing the data of various experiments that measured particle densities at ground. This could be done by introducing the concept of local age $s=s(r)$ [14], or suitably modifying the original form $[15,16,17,18]$. For ARGO-YBJ data, the LDFs (up to about 10m from the core) have been fitted with several of that parametrizations, as described in details in reference [19]. Such a systematic study showed that no one of that functions was able to fit ARGO-YBJ data in a satisfactory way. In particular, the fit with the original NKG formula did not give good results unless using $R_{M}$ values much lower than the actual Molière radius at the experimental site.

The best performance in describing both simulated and experimental data, with the minimum number of parameters and whatever the $N_{p 8}$ bin, was achieved by the use of a simplified, NKG-like, LDF defined as:

$$
\rho(r)=A \times\left(\frac{r}{r_{0}}\right)^{s^{\prime}-2}\left(1+\frac{r}{r_{0}}\right)^{s^{\prime}-4.5}
$$

where $A$ is a normalization factor, $s^{\prime}$ is the shape parameter that plays the role of the lateral age, and $r_{0}$ is a constant scale radius, which refers to the Molière radius in the classical NKG function. Both simulated and real data suggested the value $r_{0}=30 \mathrm{~m}$.

Some of these results both for Monte Carlo and for experimental data in a given $N_{p 8}$ bin are reported in Fig. 2. The left panel shows the average lateral distribution and the related fit for the p-primary event sample with $\Delta N_{p 8}=10^{4.7}-10^{5.0}$, corresponding to a typical energy $E_{p} \sim 500 \mathrm{TeV}$. 

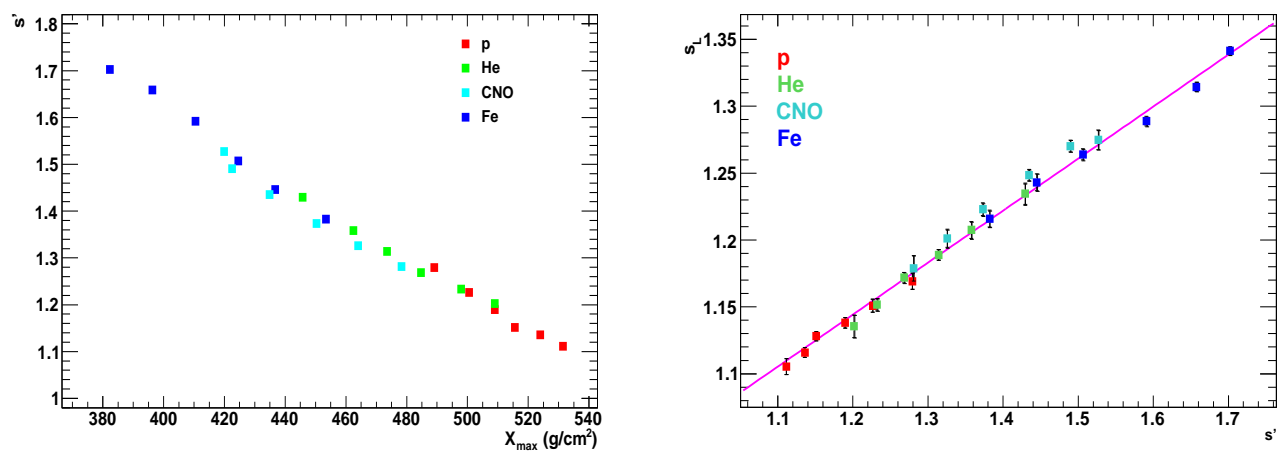

Figure 3: Left: The lateral age parameter $s^{\prime}$ resulting from the fits of the average LDF of simulated p, He, CNO group and Fe samples (in each $N_{p 8}$ bin) vs the corresponding $X_{\max }$ average values. Right: Average longitudinal age parameter $s_{L}$ vs the corresponding lateral age parameter $s^{\prime}$ resulting from the fits of the LDFs of simulated $\mathrm{p}, \mathrm{He}, \mathrm{CNO}$ group and Fe samples (in each $N_{p 8}$ bin).

In the same plot, the distribution and fit for the 'mean event' in the same $\Delta N_{p 8}$ bin, induced by iron-nucleus primaries $\left(E_{F e} \sim 1.4 \mathrm{PeV}\right)$, can also be observed. Also for experimental data, the lateral particle density profiles appear properly described by Eq. 4.1, as shown in Fig. 2 (right), where the fit to the average LDF for real events with $10^{4.7}<N_{p 8}<10^{5.0}$ is reported as an example.

\section{Shower age determination}

From the fit of the average LDF for each of the simulated primaries (p, He, CNO, Fe) and for each $N_{p 8}$ interval, it was found that, for a given primary, the fit parameter $s^{\prime}$ value decreases when $N_{p 8}$ (i.e. the energy) increases, this being due to the observation of younger (deeper) showers at larger energies. Moreover for a given range of $N_{p 8}, s^{\prime}$ increases going from proton to iron, as a consequence of a larger primary interaction cross section. Both dependencies are in agreement with the expectations, the slope $s^{\prime}$ being correlated with the shower age, thus reflecting its development stage. As a consequence, if we plot the fit $s^{\prime}$ values, for each simulated primary type and $N_{p 8}$ interval, as a function of the corresponding $X_{\max }$ average values, we obtain the correlation shown in Fig. 3 (left panel). As can be seen, the shape parameter $s^{\prime}$ depends only on the development stage of the shower, independently from the nature of the primary particle and energy. That plot expresses an important 'universality property' of the LDF of detected EAS particles in terms of the lateral shower age. Obviously, the shower-to-shower fluctuations introduce unavoidable systematics, whose effects can be anyway quantified and taken into account.

The previous outcome moreover implies that $s^{\prime}$ from the LDF fit very close to the shower axis, together with the measurement of the truncated size $N_{p 8}$, can give information on the primary particle nature, thus making possible the study of primary mass composition.

We can more deeply investigate the above described result and get further consequences: the universality property expressed by the left plot in Fig. 3 allows to decouple the detected shower signal from the primary nature thanks to the (linear) relation $s^{\prime}=s^{\prime}\left(X_{\max }\right)$, once the $s^{\prime}$ parameter value was obtained from the single event LDF fit. This really gives the possibility to identify a mass independent energy estimator, as shown in the next Section.

Moreover, as already stated, in our context the parameter $s^{\prime}$ plays the role of lateral age, so it should be strongly correlated to the longitudinal age $\left(s_{L}\right)$ of the shower. Such correlation is made 

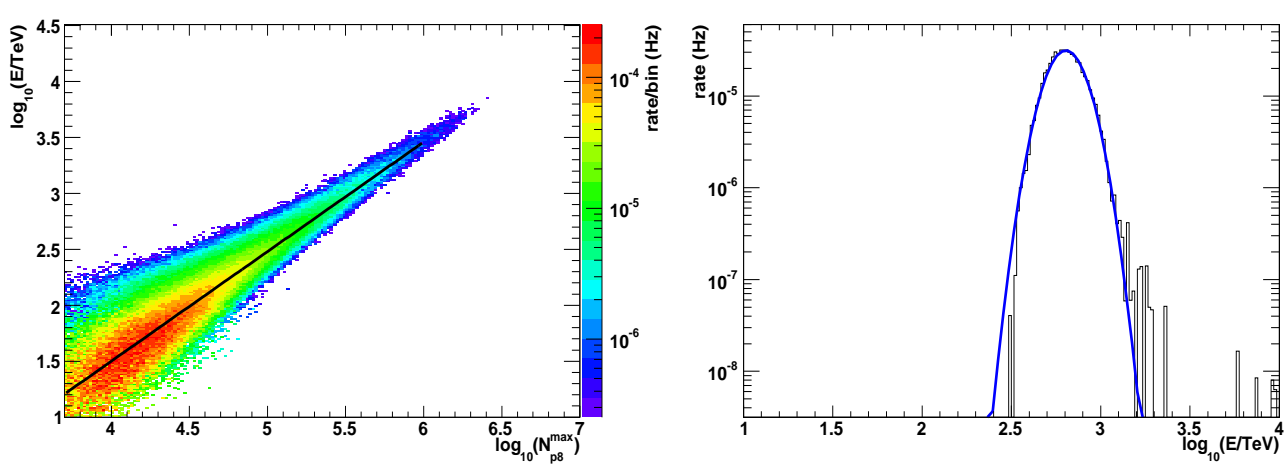

Figure 4: Left: Two-dimensional histogram of $\log _{10}(E / T e V)$ vs $\log _{10}\left(N_{p 8}^{\max }\right)$ for a simulated mixture of quasi-vertical $\left(\theta<15^{\circ}\right) \mathrm{H}, \mathrm{He}, \mathrm{CNO}$ group and Fe nuclei, in the assumption of Hörandel composition model. A linear fit is superimposed. Right: The $\log _{10}$ of energy distribution corresponding to the interval $\log _{10}\left(N_{p 8}^{\max }\right)=[5.30,5.38]$. As shown, the distribution is properly fitted by a Gaussian function.

more explicit using the classical definition of $s_{L}$ as a function of the shower maximum depth $X_{\max }$ :

$$
s_{L}=\frac{3 h_{0} \cdot \sec (\theta)}{h_{0} \cdot \sec (\theta)+2 X_{\max }}
$$

where $h_{0}$ is the detector vertical atmospheric depth, $\theta$ the shower zenith angle. Then, using the same average $X_{\max }$ values reported in Fig. 3 (left), through the previous relation we obtain the plot of Fig. 3 (right panel), which clearly demonstrates how the 'observed' lateral age $s^{\prime}$ is strictly related to the classical age $s_{L}$, and moreover a linear dependence appears appropriate. Indeed, in the same plot a linear fit has been superimposed, which gives:

$$
s_{L}=(0.389 \pm 0.005) \cdot s^{\prime}+(0.678 \pm 0.007)
$$

\section{Mass independent energy calibration}

In a first approximation, we can assume that the shower is absorbed after its maximum in the atmosphere following an exponential law:

$$
N(X)=N_{t o t}^{\max } \cdot \exp \left(-\frac{X-X_{\max }}{\lambda_{a b s}}\right)
$$

where the number $N(X)$ of particles at depth $X$ is obtained from the number of particles at maximum $N_{\text {tot }}^{\max }$, taking into account the shower maximum depth $X_{\max }$ and the absorption length in the atmosphere $\lambda_{a b s}$. It is reasonable to apply the same absorption law to the truncated size $N_{p 8}$, so as to get the correct signal at maximum, $N_{p 8}^{\max }$, inverting the function (3):

$$
N_{p 8}^{\max }=N_{p 8} \cdot \exp \left(\frac{h_{0} \cdot \sec (\theta)-X_{\max }\left(s^{\prime}\right)}{\lambda_{a b s}}\right)
$$

where $X_{\max }$ is estimated on the basis of the $s^{\prime}$ value measured from the event by event LDF fit. Equivalently, using the relation Eq. 5.1:

$$
N_{p 8}^{\max }=N_{p 8} \cdot \exp \left(\frac{3}{2} \frac{h_{0} \cdot \sec (\theta)}{\lambda_{a b s}}\left(1-\frac{1}{s_{L}\left(s^{\prime}\right)}\right)\right)
$$

which expresses $N_{p 8}^{\max }$ as a function of $s^{\prime}$, by means of the longitudinal age $s_{L}$. Then, using the relations of Eq. 5.2 and 6.3, it is possible to get the signal at maximum $\left(N_{p 8}^{\max }\right)$ for each event, on the basis of the observed truncated size $N_{p 8}$ and the shower age $s^{\prime}$ from the event by event LDF fit. 

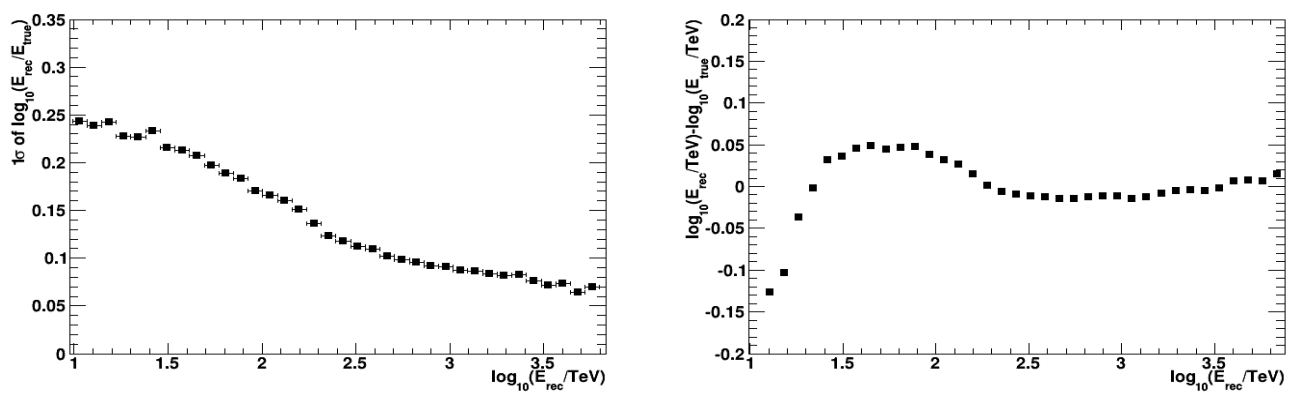

Figure 5: Energy resolution (left) and bias (right) as a function of the reconstructed energy $E_{\text {rec }}$ for quasivertical events $\left(\theta<15^{\circ}\right), \lambda_{a b s}=100 \mathrm{~g} / \mathrm{cm}^{2}$ and Hörandel model. The method was applied for $E \gtrsim 100 \mathrm{TeV}$.

The universality properties of extensive air shower development suggest that, when observed at its maximum, the shower size is only a function of the total energy, mostly independent on the primary nature [20]. This is what is really found: while the $N_{p 8}$ distributions for simulated cosmic ray showers of different species ( $\mathrm{p}, \mathrm{He}, \mathrm{CNO}, \mathrm{Fe}$ ) and same energy look quite separated, when the correction for attenuation is applied, the distributions of $N_{p 8}^{\max }$ for the different primaries in the same energy bin, as obtained using Eq. 6.3, nicely overlap. This is evident in Fig. 4 (left), where a two-dimensional histogram of the $\log _{10}\left(N_{p 8}^{\max }\right)$ quantity versus $\log _{10}(E / T e V)$ is shown for a simulated mixture of protons, $\mathrm{He}, \mathrm{CNO}$ group and $\mathrm{Fe}$, weighted by the flux model proposed by Hörandel. Very similar results are obtained using other composition models. Monte Carlo events have been selected with the same quality cuts of real data and zenith angle within $15^{\circ}$. The twodimensional histogram has been sliced in 0.08 width bins of $\log _{10}\left(N_{p 8}^{\max }\right)$ and for each slice the $\log _{10}$ of energy distribution has been obtained. All the $\log _{10}$ of energy distributions have been properly fitted by a Gaussian (see right panel of Fig. 4 for an example) in order to estimate the mean value of $\log _{10}$ of energy. Over the plot in Fig. 4 (left), a stright line is superimposed, which represents the relationship between the logarithm of truncated size at maximum and the fitted mean of the energy distribution. Such relationship is well described by a linear fit:

$$
\log _{10}(E / T e V)=a \cdot \log _{10}\left(N_{p 8}^{\max }\right)+b
$$

being $\mathrm{a}=(0.98 \pm 0.01)$ and $\mathrm{b}=(-2.42 \pm 0.05)$.

The energy resolution, defined as one sigma of the distribution of the quantity $\log _{10}\left(E_{\text {rec }} / E_{\text {true }}\right)$ (being $E_{\text {true }}$ the true energy of the simulated event and $E_{\text {rec }}$ the energy value reconstructed from Eq. 6.4), has been evaluated from Monte Carlo simulation. A value of $0.10-0.15$ in $\log _{10}(E / T e V)$ has been obtained in the energy range starting from $10^{4} \mathrm{eV}$ (where the procedure was applied to the data), getting better with energy, as shown in Fig. 5 (left), where the resolution is plotted as a function of the reconstructed energy. Moreover, the energy reconstruction bias, defined as the difference $\log _{10}\left(E_{\text {rec }} / \mathrm{TeV}\right)-\log _{10}\left(E_{\text {true }} / \mathrm{TeV}\right)$, has been found within 0.05 in $\log _{10}(E / T e V)$, in the energy range useful for ARGO-YBJ measurements, as reported in Fig. 5 (right).

The absorption length $\lambda_{a b s}$ has been determined by optimizing the energy resolution and bias for a fixed energy value. The value $\lambda_{a b s}=100 \mathrm{~g} / \mathrm{cm}^{2}$ satisfies both the request of resolution less than 0.1 in $\log _{10}(E / T e V)$ and bias within 0.05 for the whole energy interval $100 \mathrm{TeV}-10 \mathrm{PeV}$. That value is compatible with a previous ARGO-YBJ measurement [6].

Such energy calibration method, based on the use of the lateral age concept, allowed the measurement of all-particle CR spectrum from ARGO-YBJ data, in agreement with other experimental 
results [21]. The light $(\mathrm{p}+\mathrm{He})$ component spectrum was also measured, through the primary mass selection capability offered by the same parameter $s^{\prime}$, as extensively discussed in [21].

\section{Conclusions}

A detailed study of the lateral particle distribution (LDF) around the axis of the quasi-vertical extensive air showers detected by the ARGO-YBJ experiment has been performed. The analysis of data triggering the RPC charge readout system allowed to explore a wide range of particle density, from few particles $/ \mathrm{m}^{2}$ up to several $10^{4} / \mathrm{m}^{2}$ very near to the core. Applied to simulated data, this study showed how the slope of this LDF distribution, expressed by a lateral age parameter $s^{\prime}$, gives information on the longitudinal shower development. Moreover, it demonstrated the existence of an important universality property of the shower development stage, when expressed in terms of $s^{\prime}$, with respect to the primary nature. Assuming an exponential absorption of the shower particles after the maximum, the above outcomes allowed to obtain a powerful mass independent primary energy estimation for the ARGO-YBJ events, only based on the truncated size of detected charged particles $\left(N_{p 8}\right)$ and the $s^{\prime}$ parameter value from the LDF fit within a distance of $8 \mathrm{~m}$ from the core. This method allowed ARGO-YBJ to successfully measure both all-particle and ( $\mathrm{p}+\mathrm{He}$ ) CR spectra.

\section{References}

[1] K. Greisen, Progress in Cosmic Ray Physics, Vol.III (NH Publishing Co, Amsterdam, 1956)

[2] K. Kamata, J. Nishimura, Prog. Theor. Phys. Supplement No.6 (1958) pp. 93-155.

[3] R.K. Dey et al., J. Phys. G: Nucl. Part. Phys. 39, 085201 (2012)

[4] A D'Amone et al. (for ARGO-YBJ Collaboration), ISVHECRI-2012 proceedings.

[5] I. De Mitri (for ARGO-YBJ Collaboration), J. Phys.: Conf. Ser. 375052027 (2012).

[6] C. Bacci et al. (ARGO-YBJ Collaboration), Astropart. Phys. 17, 151 (2002)

[7] G. Aielli et al. (ARGO-YBJ Collaboration), NIM A 562, 92 (2006)

[8] B. Bartoli et al. (ARGO-YBJ Collaboration), Astropart. Phys. 67, 47 (2015)

[9] D. Heck et al., Report FZKA 6019, Forschungszentrum Karlshruhe (1998).

[10] S. Ostapchenko, Nucl. Phys. B, 151143 (2006)

[11] J.R. Hörandel, Astropart. Phys., 19193 (2003)

[12] GEANT - Detector Description and Simulation Tool, CERN Program Library, W5013 (1993).

[13] A. D'Amone et al. (for ARGO-YBJ Collaboration), Proc. 33rd ICRC (2013), paper id. 779.

[14] J. N. Capdevielle, F. Cohen, J. Phys. G: Nucl. Part. Phys. 31 (2005) 507-524.

[15] W.D. Apel et al., Astrop. Phys. 24 (2006) 467-483.

[16] M. Takeda et al., Astrop. Phys. 19 (2003) 447-462.

[17] J. Linsley et al., J. Phys. Soc. Japan 17 (1962) Suppl. A-III 91.

[18] R.I. Raikin et al., Nucl. Phys. B (Proc. Suppl.) 175-176 (2008) 559-562.

[19] P. Bernardini et al. (for ARGO-YBJ Collaboration), Proc. 33rd ICRC (2013), paper id. 781.

[20] J. Blümer et al., Progr. in Part. and Nucl. Phys. 63, 293 (2009).

[21] A. D'Amone et al. (for ARGO-YBJ Collaboration), Proc. of this Conference, paper id. 917. 